\title{
THE DYNAMIC MERGE: USING TRAFFIC VOLUME BASED SIGNING TO IMPROVE WORKZONE THROUGHPUT
}

\author{
Starla M. Weaver ${ }^{1}$, Michelle Arnold ${ }^{2}$, Tracy Gonzalez ${ }^{1}$, Stacy A. Balk ${ }^{1}$ \\ ${ }^{1}$ Leidos, McLean, VA, USA \\ ${ }^{2}$ Federal Highway Administration, US Department of Transportation \\ McLean, VA, USA \\ Email: starla.weaver@leidos.com
}

\begin{abstract}
Summary: Roadwork that results in a lane closure can reduce both the safety and efficiency of a roadway. The dynamic merge is a form of merge control designed to mitigate the potential hazards of lane closures by customizing the merge environment to suit the current level of traffic. When traffic is light, early merge signs encourages drivers to merge into the open lane prior to queue formation. When traffic is heavy, late merge signs encourages drivers to remain in the closed lane for as long as possible. The current study used a driving simulator to assess the independent effects of traffic volume and dynamic merge messaging on merge location and traffic throughput. Merge location was influenced by merge environment, such that drivers in the early merge condition merged earlier than those in the late merge condition regardless of traffic volume. In addition, when traffic was heavy, participants in the late merge condition passed through the work zone more quickly than those in the early merge condition. The results are suggestive of the beneficial effects of the late merge on traffic throughput and the effectiveness of the dynamic merge messaging in influencing merging behavior.
\end{abstract}

\section{OBJECTIVES}

Lane reductions reduce roadway capacity. As a result, it is not uncommon for vehicle queuing to occur prior to a lane closure. This queuing can be a safety hazard. Drivers traveling at high speeds that suddenly encounter slower moving traffic are at risk of queue-end-collisions and may have difficulty safely merging into the slower moving queue (Ullman, Iragavarapu, \& Brydia, 2016). This situation can also lead to aggressive driving. Drivers in the open lane may attempt to slip past those already in the queue, while drivers in the closed lane may actively try to prevent merging by straddling both lanes or keeping pace with incoming vehicles to block them from entering the open lane (McCoy \& Pesti, 2001).

The advance warning area is the portion of roadway where travelers are informed about the upcoming work zone. This area is designed to reduce the potential hazards associated with lane reductions by instructing drivers on how to prepare for the upcoming lane closure. The Manual on Uniform Traffic Control Devices (MUTCD) specifies a series of signs that should be used within the advance warning area (National Advisory Committee on Uniform Traffic Control Devices, 2009). Local agencies can choose to supplement these standard signs with additional signing to create merge environments aimed at increasing roadway safety and efficiency. Three merge environments are considered below. 


\section{Early Merge}

The early merge is designed to prevent the potential safety concerns associated with merging through signs that encourage drivers to merge early, that is before the queue. Early merge environments have been found to reduce cut-ins and decrease anger among queueing drivers (Nemeth \& Rouphail, 1982; Tarko, Kanipakapatnam, \& Wasson, 1998). As a result, the early merge is considered a successful way of increasing the safety of a lane reduction. However, because this strategy increases queue length, it also functionally extends the length of the lane closure, thus reducing the capacity of the roadway and negatively affecting traffic throughput (Mousa, Rouphail, \& Azadivar, 1990; Tarko et al., 1998). This negative effect is particularly severe when traffic volumes are high (McCoy \& Pesti, 2001).

\section{Late Merge}

The late merge encourages drivers to remain in both lanes for as long as possible. Within a late merge, signs warn drivers about the upcoming lane closure but encourages them to remain in their lane as they approach the merge location. An additional sign just prior to the lane reduction indicates the appropriate merge point (United Research Services, 2004). By encouraging use of both lanes for as long as possible, late merge environments significantly increases traffic throughput (Grillo, Datta, \& Hartner, 2008; Kang, Chang, \& Paracha, 2006; Radwan, Zaidi, \& Harb, 2011). Additionally, proponents of the late merge suggest that the aggressive behavior sometimes seen by queuing drivers is primarily motivated by the belief that drivers who continue to use the closed lane are "breaking the rules" or "skipping the line" (McCoy \& Pesti, 2001). If signs indicate that use of both lanes is appropriate, then aggressive actions against vehicles that use the closed lane should be reduced.

Late merge environments increase traffic throughput, particularly when traffic volumes are high (Grillo et al., 2008; Kang et al., 2006; Radwan et al., 2011). However, potential safety concerns about this merge strategy exist, particularly when traffic volumes are low. The late merge specifies a single location for merging just prior to the lane closure. When traffic is light, vehicles may still be traveling at high speeds when they reach this point, such that vehicles in the closed lane may have difficulty merging safely within this small merge area. This safety concern is exacerbated by the potential confusion caused by the conflicting messages provided by late merge messaging and the standard signs recommended by the MUTCD (United Research Services, 2004).

\section{Dynamic Merge}

The dynamic merge is a flexible strategy that customizes the merge environment to suit the specific level of traffic currently occurring on the roadway (McCoy \& Pesti, 2001). Under this system, dynamic signs are paired with devices that monitor the volume or speed of traffic in the advance warning area. When traffic is light, the early merge system is engaged and drivers are encouraged to merge into the open lane early. When traffic is heavy the late merge system is engaged and drivers are encouraged to merge farther downstream. Studies examining the effectiveness of dynamic merge environments have found changes in the position of lane merges, reduction in queue lengths, and increases in traffic throughput in response to the dynamic merge (Harb et al., 2009; Radwan et al., 2011; United Research Services, 2004). 
Dynamic merge environments combine the potential safety benefits of the early merge system during light traffic with the potential throughput benefits of the late merge system during heavy traffic (McCoy \& Pesti, 2001). However, because the dynamic merge system changes as a function of traffic volume, it is difficult to confirm that the difference in merge location of drivers exposed to each merge environment is a direct result of the merge environment itself and not a result of the corresponding traffic levels. Previous research has found that congestion levels influence both route and lane choice (Pan \& Khattak, 2008; Xuan \& Kanafani, 2014). In fact, traffic volume has been argued to serve as a greater determinant of traveler behavior than information presented on road signs (Xuan \& Kanafani, 2014). It is therefore possible that the changes in behavior seen in response to the dynamic merge are actually a product of the same traffic levels that prompt the change in messaging, rather than a response to the messaging itself. Given the effort and expense required to create a dynamic merge environment, it is prudent to ensure that the potential benefits of this system are not simply a biproduct of traffic volume. In order to investigate this possibility, and ascertain the true impact of dynamic merge messaging, the current study used a driving simulator to manipulate both merge environment and traffic volume independently.

\section{METHOD}

\section{Participants}

Participants were 120 licensed drivers from the greater Washington, DC, metropolitan area. All drivers were over the age of 18 .

\section{Apparatus}

The driving simulator used for this study consists of a compact sedan mounted on a 6-degree of freedom motion base. A 200-degree screen with a radius of 8.9 feet surrounds the simulator. Three projectors with resolutions of $2048 \times 1536$ pixels, project stimuli onto the screen. The design eye point of the simulator is 9.5 feet directly in front of the driver.

\section{Design}

The study included two independent variables. Merge environment was manipulated betweensubjects to be either early or late. Messaging appeared on three portable message signs located on the right side of the roadway. The specific messages presented during each merge environment are displayed in Figure 1. Traffic volume was also manipulated between-subjects to be either light (5.82 vehicles per minute) or heavy ( 8.92 vehicles per minute). Dependent variables were merge location, vehicle speed, and the time required to clear the advance warning area.

\section{Procedure}

Participants first completed a brief practice drive to familiarize themselves with the simulator. They practiced acceleration, braking, changing lanes, and exiting the highway to become comfortable with the speed and steering dynamics of the simulation vehicle.

Participants then completed the simulated scenario beginning in the left lane of a four-lane divided highway with a speed limit of $65 \mathrm{mph}$. After traveling for four miles, participants passed below an overhead variable message sign (VMS) indicating that an event that was impacting 
traffic was occurring on the road ahead. Three and a half miles downstream from the VMS, participants entered the advance warning area. Figure 2 shows sign placement within the area, which started 7.5 miles into the simulation. Consistent with the recommendations of the MUTCD, static message signs appear on both sides of the roadway at three locations. Sign w201 (ROAD WORK 1 MILE) was positioned at mile 8, one mile prior to the lane closure; sign w20-5 (LEFT LANE CLOSED 1/2 MILES) at mile 8.5, 1.5 miles prior to the lane closure; and sign w4-2 (the merge symbol) at mile 8.8, 0.2 miles prior to the lane closure. An arrow board marked the beginning of the taper, where traffic barrels began to close the left lane. Following the taper, the left lane was blocked by the event mentioned on the overhead VMS.

The design of the dynamic merge area was a modified version of the design used by Kang et al. (2006). Three portable message signs were positioned on the left side of the road at 1.5 (P3), 0.4 (P2), and $0.1(\mathrm{P} 1)$ miles prior to the lane closure. The specific messages on these signs varied by condition (see Figure 1) to create an early or late merge environment.

\begin{tabular}{|c|c|c|c|c|c|c|}
\hline & \multicolumn{2}{|c|}{ P3 } & \multicolumn{2}{|c|}{ P2 } & \multicolumn{2}{|c|}{ P1 } \\
\hline $\begin{array}{l}\text { Early } \\
\text { Merge }\end{array}$ & $\begin{array}{l}\text { ROAD } \\
\text { WORK } \\
\text { AHEAD }\end{array}$ & $\begin{array}{l}\text { LEFT LANE } \\
\text { CLOSED }\end{array}$ & $\begin{array}{l}\text { LEFT LANE } \\
\text { CLOSED }\end{array}$ & $\begin{array}{c}\text { MERGE } \\
\text { RIGHT }\end{array}$ & & \\
\hline $\begin{array}{l}\text { Late } \\
\text { Merge }\end{array}$ & $\begin{array}{l}\text { SLOW } \\
\text { TRAFFIC } \\
\text { AHEAD }\end{array}$ & $\begin{array}{l}\text { USE BOTH } \\
\text { LANES }\end{array}$ & $\begin{array}{l}\text { USE BOTH } \\
\text { LANES }\end{array}$ & $\begin{array}{l}\text { TO MERGE } \\
\text { POINT }\end{array}$ & $\begin{array}{l}\text { TAKE } \\
\text { YOUR } \\
\text { TURN }\end{array}$ & $\begin{array}{c}\text { MERGE } \\
\text { HERE }\end{array}$ \\
\hline
\end{tabular}

Figure 1. Messages displayed within each merge environment

Simulated traffic traveled in both lanes through the drive. The behavior of the simulated traffic was controlled by a microscopic traffic flow simulation software. To control for the potential social influences of traffic, the parameters that guided the simulated traffic's behavior, including lane usage patterns, were consistent across all conditions. Traffic volume was manipulated to be either heavy or light. In the light traffic condition, participants could pass through the lane reduction with minimal delay. Within the heavy traffic condition, participants encountered queuing prior to the lane closure.

After driving the scenario, participants completed a brief questionnaire that including rating their stress level during the drive on a scale from one to six. Then participants completed a legibility drive to determine a maximum legibility distance for each participant. The scenario used for this drive was the same as that used during the larger experiment except a novel VMS message was displayed. Participants were asked to drive slowly toward the VMS and stop as soon as they could read the VMS message, with the goal of trying to read the message from as far away as possible. When participants reached their maximum legibility distance they placed the vehicle in park and the distance was recorded. Finally, participants were debriefed and paid for their time.

\section{Analyses}

Generalized estimating equation (GEE) models were used to test the associations between variables. All independent variables were included in the initial model. Insignificant effects were removed and the models were rerun until the effects remaining were all significant. Participants whose final merge occurred before they were within view of the advance warning area were 
excluded from analysis. This point was defined individually for each participant by subtracting that participant's maximum legibility distance, found during the legibility drive, from the position of $\mathrm{p} 3$ ( 7.5 miles). The final analyses included data from 78 participants: 18 participants in the early merge, heavy traffic condition; 21 in the early merge, light traffic condition; 25 in the late merge, heavy traffic condition; and 14 in the late merge, low traffic condition. The high levels of participant loss is a limitation of the current study. Nevertheless, the proportion of drivers excluded did not vary as a function of condition, $\chi^{2}(1)=2.54, p=.111$, and is therefore unlikely to have affected the current pattern of findings.

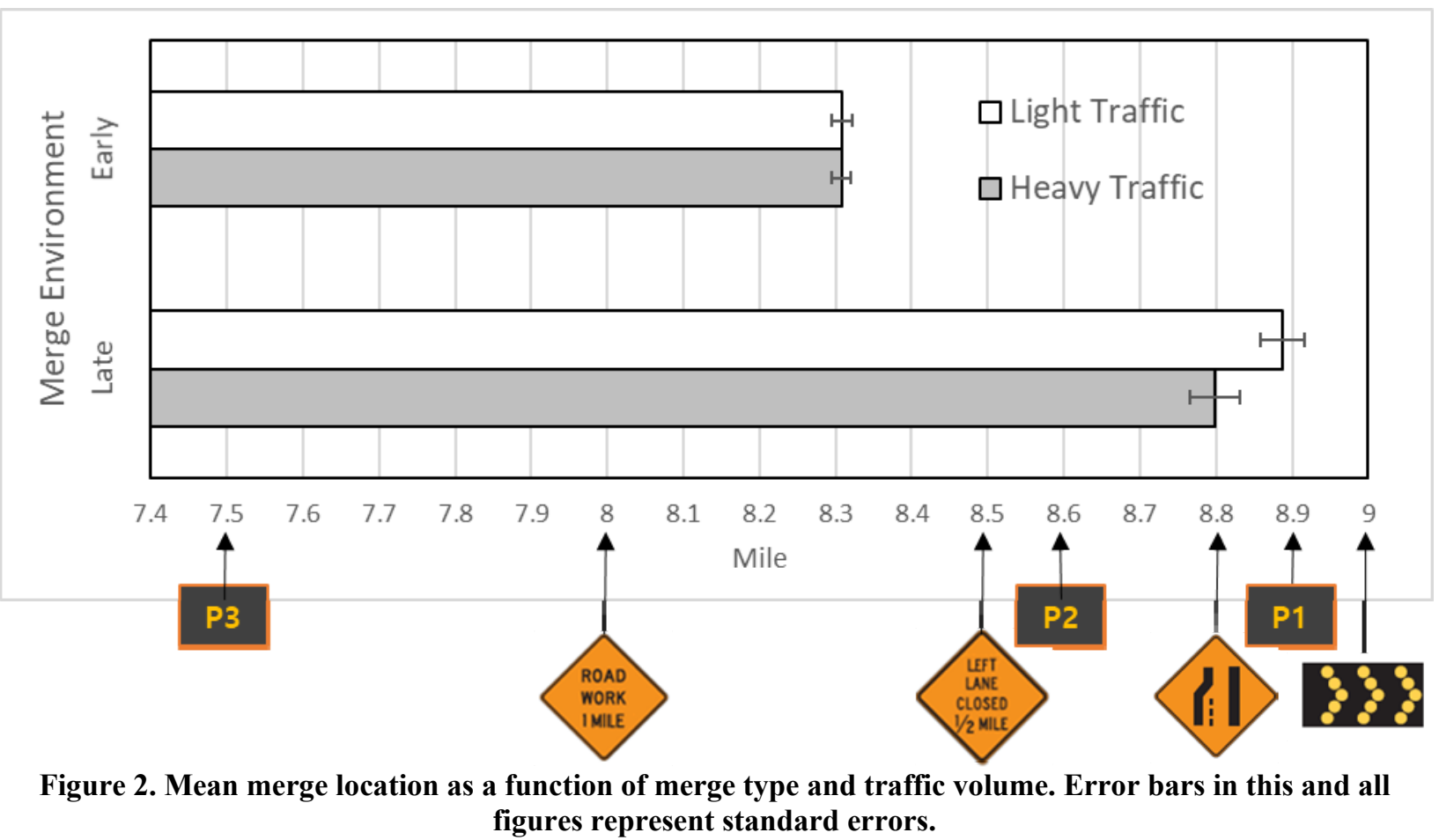

\section{RESULTS}

Merge location was assessed as a function of merge environment and traffic volume. An effect of merge environment was found, $\chi^{2}(1)=25.36, p<.001$. As is displayed in Figure 2, participants exposed to the early merge environment merged earlier $(M=8.31$ miles) than participants exposed to the late merge environment ( $M=8.83$ miles). Merge location did not vary as a function of traffic volume.

The speed participants drove within the advanced work zone was also assessed. As expected, participants in the light traffic condition drove significantly faster $(M=50.81 \mathrm{mph})$ than participants in the heavy traffic condition $(M=26.78 \mathrm{mph}), \chi^{2}(1)=24.03, p<.001$. Merge environment did not influence speed.

Traffic volume also influenced the amount of time required to travel through the advance warning area, $\chi^{2}(1)=100.72, p<0.001$. Those in the light traffic condition traveled through the warning area more quickly $(M=121 \mathrm{sec}$. $)$ than those in the heavy traffic condition $(M=235 \mathrm{sec}$. $)$. An interaction between traffic volume and merge environment was also found, $\chi^{2}(1)=26.20$, $p=0.029$. As displayed in Figure 3, when traffic was light all participants moved through the 
advance warning area quickly. However, when traffic was heavy, participants cleared the area more quickly when exposed to the late merge environment than when exposed to the early merge environment.

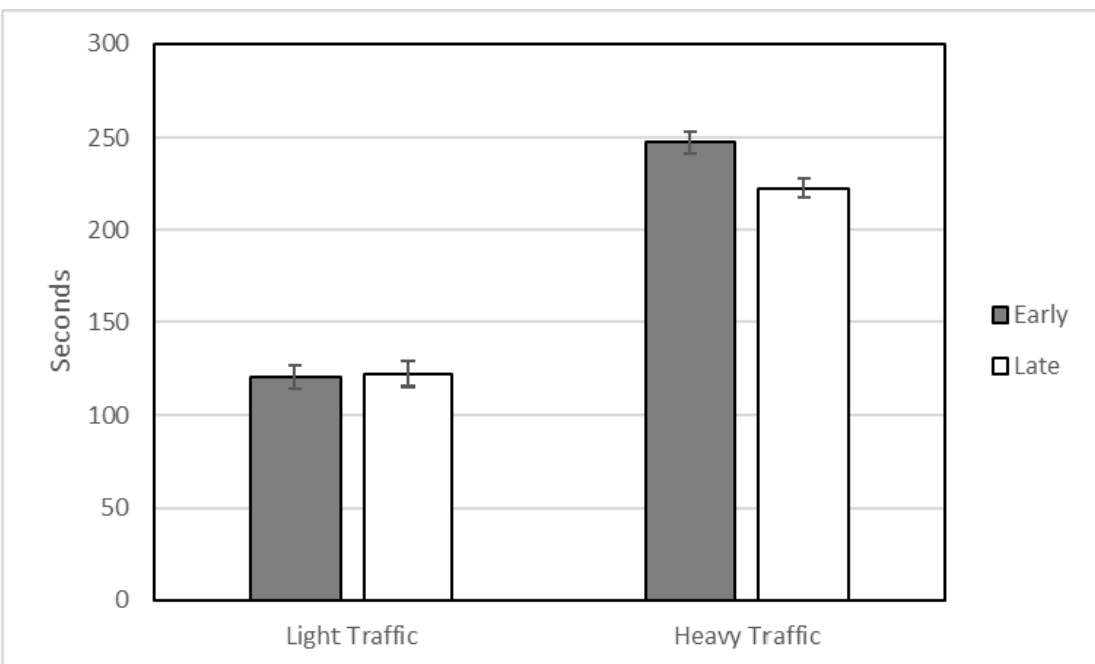

Figure 3. Mean time spent driving through the advanced warning area as a function of merge type and traffic volume.

Post-drive stress levels were assessed on a scale of 1 to 6 . Overall stress levels were low $(M=1.58)$ and did not vary as a function of traffic volume or merge environment, $p>0.05$.

\section{CONCLUSIONS}

The current study assessed the independent effects of traffic volume and dynamic merge environment on merge position and time to clear the advance warning area prior to a lane closure. Across both light and heavy traffic conditions, participants in the early merge condition merged into the open lane earlier than participants in the late merge condition. This difference in merge location was independent of the lane usage patterns of the other vehicles on the roadway, which did not vary between simulations. The results build on past dynamic merge research by demonstrating that the changes in traffic patterns seen in response to early and late merge environments in previous research (McCoy \& Pesti, 2001; United Research Services, 2004) are a result of dynamic merge signs' ability to influence merge location, rather than simply a biproduct of traffic volume.

The study also found that when traffic was heavy, participants within the late merge condition cleared the advance warning area sooner than those in the early merge condition. The results are suggestive of the beneficial effects of late merge messaging on traffic throughput. By encouraging drivers to use both lanes until the merge point, the late merge environment maximizes road use and prevents excessive queuing prior to the lane closure (Harb et al., 2009; Radwan et al., 2011). The results support using traffic volume based signing to increase roadway safety and efficiency during a lane closure.

\section{ACKNOWLEDGMENTS}

This work was funded by Federal Highway Administration Contract DTFH61-13-D-00024. 


\section{REFERENCES}

Grillo, L., Datta, T., \& Hartner, C. (2008). Dynamic late lane merge system at freeway construction work zones. Transportation Research Record: Journal of the Transportation Research Board, 2055, 3-10.

Harb, R., Radwan, E., Ramasamy, S., Abdel-Aty, M., Pande, A., Shaaban, K., \& Putcha, S. (2009). Two simplified dynamic lane merging system (SDLMS) for short term work zones. Paper presented at the Transportation Research Board 88th Annual Meeting.

Kang, K.-P., Chang, G.-L., \& Paracha, J. (2006). Dynamic late merge control at highway work zones: evaluations, observations, and suggestions. Transportation Research Record, 1948(1), 86-95.

McCoy, P., \& Pesti, G. (2001). Dynamic late merge-control concept for work zones on rural interstate highways. Transportation Research Record: Journal of the Transportation Research Board(1745), 20-26.

Mousa, R. M., Rouphail, N. M., \& Azadivar, F. (1990). Integrating microscopic simulation and optimization: Application to freeway work zone traffic control. Transportation Research Record, 1254, 14-25.

National Advisory Committee on Uniform Traffic Control Devices. (2009). Manual on uniform traffic control devices for streets and highways. Washington DC: FHWA Department of Transportation,.

Nemeth, Z. A., \& Rouphail, N. M. (1982). Lane closures at freeway work zones: Simulation study. Transportation Research Record, 869, 19-25.

Pan, X., \& Khattak, A. (2008). Evaluating traveler information effects on commercial and noncommercial users. Transportation Research Record: Journal of the Transportation Research Board, 2086, 56-63.

Radwan, E., Zaidi, Z., \& Harb, R. (2011). Operational Evaluation of Dynamic Lane Merging In Work Zones with Variable Speed Limits. Procedia-Social and Behavioral Sciences, 16, 460-469.

Tarko, A. P., Kanipakapatnam, S. R., \& Wasson, J. S. (1998). Modeling and Optimization of the Indiana Lane Merge Control System on Approaches to Freeway Work Zones, Part I. Lafayette, IN: Joint Transportation Research Program

Ullman, G. L., Iragavarapu, V., \& Brydia, R. E. (2016). Safety Effects of Portable End-of-Queue Warning System Deployments at Texas Work Zones. Transportation Research Record: Journal of the Transportation Research Board, 2555, 46-52.

United Research Services. (2004). Evaluation of 2004 dynamic late merge system Minnesota Department of Transportation

Xuan, Y. E., \& Kanafani, A. (2014). Evaluation of the effectiveness of accident information on freeway changeable message signs: A comparison of empirical methodologies. Transportation Research Part C: Emerging Technologies, 48, 158-171. 